\title{
Technologie et âge avancé au Japon
}

\author{
Yasuyuki Gondo
}

La population des personnes âgées a augmenté au cours des dernières décennies au Japon, une évolution démographique importante qui a fourni de nouvelles opportunités commerciales aux entreprises. Les technologies et services innovants pour les personnes âgées se sont développés et certains sont déjà en cours d'utilisation. De plus, la révolution technologique semble promettre à la future société super-vieillissante de réussir à bien vieillir. Le présent rapport fournit une vue d'ensemble des technologies actuellement appliquées auprès des personnes âgées et donne quelques exemples de nouvelles technologies qui se développent au Japon.

\section{Introduction}

Le Japon est un pays précurseur dans les progrès accomplis dans le domaine du vieillissement de la société (Muramatsu and Akiyama, 2011). La présentation de l'expérience japonaise pourrait être une référence utile pour d'autres pays. Dans le domaine technologique en particulier, de nombreux services opérationnels ont déjà été introduits ou sont en cours de développement aujourd'hui. Pour traiter ces questions, j'ai séparé la technologie pour les personnes âgées en trois dimensions fonctionnelles : compensation, communication et promotion de la santé. Ici, la compensation est synonyme d'aide ou de regain de la fonction à travers l'utilisation de la technologie comme les appareils auditifs par exemple. La communication est synonyme de lien entre les personnes et d'amélioration du bien-être. La promotion de la santé indique le maintien de la santé à travers la technologie de communication à l'instar de la surveillance de l'activité à l'aide de montres intelligentes (smartwatch).

\section{Technologie de compensation}

Une étude a révélé que les personnes âgées au Japon se plaignaient de problèmes d'audition dans les mêmes proportions que les personnes âgées en Europe (environ 10\%). Toutefois, les utilisateurs d'appareils auditifs y sont moins nombreux (14\%) que dans les pays occidentaux (24 à 41\%) (JapanTrak 2012). Alors que les dispositifs à porter dans l'oreille ne sont pas populaires, d'autres types de dispositifs comme les prothèses à conduction osseuse et leur application aux téléphones à conduction osseuse connaissent un engouement certain. D'autres appareils uniques en leur genre ont également été commercialisés. Le dispositif le plus populaire est un système d'extension pour hautparleur pour téléviseurs qui permet aux personnes âgées d'écouter le son de la télévision près d'eux sans augmenter le volume du téléviseur. 
Pour la technologie de diffusion, un système de conversion du débit de parole en temps réel, développé il y a de cela plus de 10 ans, pourrait recevoir plus d'attention à l'avenir. Ce système a été inventé par le NHK Broadcasting Culture Research Institute (Institut de recherche de diffusion de la culture) et les radios équipées de ce mécanisme ont été commercialisées.(https://www.nhk.or.jp/strl/publica/bt/en/pr0013.pdf). Ce produit est littéralement adapté aux exigences de notre époque. Les fidèles auditeurs du programme de minuit diffusé par NHK sont des personnes âgées depuis son lancement en 1989. Aujourd'hui, il emploie de nombreux DJ retraités (âgés de 49 à 74 ans avec une moyenne d'âge de 64 ans) et diffuse les contenus préférés des auditeurs âgés. Ce fait est également révélateur du fait que la demande latente concernant la compensation auditive est élevée chez les personnes âgées. L'une des raisons principales de nonutilisation des appareils auditifs est la gêne occasionnée par le port de dispositifs mal adaptés. Des services plus complexes et intégrés sont nécessaires pour la compensation auditive.

Des dispositifs d'assistance pour la compensation de mobilité sont importants pour pouvoir vivre de manière autonome. Si vous alliez faire les courses dans les centre-villes japonais, vous reconnaîtriez facilement ces femmes âgées qui poussent des déambulateurs appelés «voiture argent». Une variété de dispositifs de ce genre aux couleurs bigarrées sont vendues en magasin. Les voitures argent type possèdent des compartiments de courses et des bancs provisoires pour se reposer.

Les véhicules électriques sont populaires auprès des personnes les plus faibles. Les premiers produits commerciaux sont arrivés dans les magasins dans les années 1980. Maintenant, deux types de véhicules sont vendus : avec manette et poignée de commande. Il est intéressant de constater que le volume d'expédition a chuté de 2002, où il a connu un pic (33.477), à 2014 (19.438). L'une des explications possibles pour cette perte de popularité réside dans le concept «utilisez-le ou vous le perdrez» qui se répand au Japon. Non seulement les personnes âgées mais également les membres de la famille, qui sont des soignants potentiels au Japon, s'inquiètent de l'augmentation de la perte de fonction due à la dépendance à cet appareil pratique. Récemment, des fauteuils roulants combinés à des pédales de type vélo ont été commercialisés (http://www.starlinxpedalchair.com/history.html). L'avantage de ce véhicule est qu'il utilise le mouvement des jambes pour avancer même si l'utilisateur est assis dans le fauteuil. Des robots de technologie plus avancée qui réhabilitent la fonction perdue ont été également développés (http://www.cyberdyne.jp/english/products/HAL/index.html). L'apport d'un soutien minimum à la fonction déclinante serait une tendance de la future technologie de compensation.

Le micro-véhicule électrique est le candidat favori pour le transfert à courte distance à l'extérieur du domicile. Ce type de véhicule a l'avantage d'avoir de faibles taux d'émissions et d'être pratique.

Cependant, un permis de conduire est exigé pour les utiliser actuellement. Le gouvernement du pays a lancé un nouvel examen de renouvellement de permis pour les conducteurs âgés de 75 ans et plus afin 
d'éviter des accidents. En considérant les principaux utilisateurs de ce véhicule, la gestion de l'autorisation de conduire constituera un problème majeur. La mise en œuvre de systèmes de conduite automatique serait indispensable pour l'évolution future.

\section{Communication}

L'augmentation du nombre de personnes âgées vivant seules constitue également un problème au Japon. Le gouvernement japonais estime à 6 millions le nombre de personnes âgées vivant seules en 2015, un chiffre qui est susceptible de passer à 7,6 millions en 2035. Un documentaire télévisé diffusé en 2005 sur le «kodoku shi», le décès d'une personne qui vit seule et meurt chez elle sans que personne ne le remarque avant très longtemps, a eu un impact sur notre société. Le livre blanc sur le vieillissement de la société en 2015 stipule que 45\% des personnes âgées vivant seules ne pensent pas que le «kodoku shi» est le problème des autres. On considère qu'un appareil de communication qui connecte les personnes âgées et leur famille ou la société empêche l'isolement social et réduit le «kodoku shi».

Nous possédons une longue expérience d'utilisation de la technologie concernant ce problème. Les systèmes d'appels d'urgence ciblant les personnes âgées ont été développés dans les années 1970. C'est un système de communication de type actif, informant des conditions d'urgence en utilisant des terminaux sans fil de type pendentifs ou des boutons d'urgence installés sur le mur. En l'an 2000, plus de $95 \%$ des gouvernements locaux ont fourni ce service d'une manière ou d'une autre. Toutefois, plusieurs articles ont signalé que le nombre actuel d'utilisateurs était inférieur à $10 \%$ des foyers âgés, les personnes âgées hésitant fréquemment à utiliser ce système même s'ils l'ont.

Le système d'assistance en ligne «mimamori» (protection) qui utilise une bouilloire électrique pour surveiller les activités des individus a été lancé pour la première fois sur le marché en 2001 en combinaison avec un produit utilisé dans la vie de tous les jours. Ce service a été développé par une société de fabrication de bouteilles sous vide (http://www.zojirushiworld.com/activities/amenities.html). Ce système contrôle l'utilisation d'une bouilloire électrique par des résidents et permet de contrôler les personnes âgées à travers la communication passive. Cette communication passive était une idée brillante à l'époque au vu de la grande tendance des personnes âgées hésitant à utiliser la communication d'urgence active. Malheureusement, le nombre d'utilisateurs a chuté, pasant de 7600 au début à 3600 aujourd'hui. Le concept de communication passive survit et est l'héritage de dispositifs plus sophistiqués comme contrôler l'électricité ou l'utilisation du gaz de ville, détecter les mouvements ou des informations biologiques comme les capteurs de respiration dans la maison.

Récemment, des prototypes de robots humanoïdes de communication mettant en œuvre des fonctions de communication ont été lancés sur le marché par différentes sociétés. Elles envisagent de positionner les robots comme interfaces clé pour des services complets allant de la surveillance à la promotion de la santé. À ce jour, en raison de questions de fiabilité, les services de surveillance et de promotion de 
la santé fournis par ces robots humanoïdes sont en phase d'essai et non encore commercialisés.

Pour le moment, le produit qui rencontre le plus de succès sur le plan commercial dans la dimension de communication est le Paro. Le Paro est un bébé phoque robot créé pour produire des effets thérapeutiques (http://www.parorobots.com/). Bien que la communication langagière soit impossible, les effets thérapeutiques ont été scientifiquement prouvés. De nombreux centres médicalisés au Japon et dans d'autres pays ont déjà utilisé Paro, principalement pour la stabilisation mentale des personnes démentes. Telenoid (http://www.geminoid.jp/projects/kibans/res/Telenoid-resources.html) est également un robot de communication inventé au Japon. Il est intéressant de noter que les personnes démentes montrent une intimité avec ce robot atypique dans la phase d'essai au Danemark.

\section{Promotion de la santé}

La dimension de promotion de la santé est un segment croissant de la technologie mais pas uniquement pour les personnes âgées. Cette année, une large étude épidémiologique sur l'incidence de la démence a été lancée avec l'utilisation d'un capteur d'activité de type bracelet sur 1000 personnes. Seront étudiés dans ce projet, l'activité quotidienne, le sommeil et la fréquence de conversation contrôlée par les bracelets et leur corrélation avec les niveaux de bêta-amyloïde cérébrale (http://www.pcworld.com/article/2971792/gadgets/toshiba-rolls-out-activity-trackers-for-seniors.html). En plus des dispositifs de contrôle innovants, les technologies courantes ont été reconsidérées. Quelques années plus tôt, les médias déclaraient que les personnes âgées étaient en passe de constituer la majorité des personnes fréquentant les centres de divertissement (http://edition.cnn.com/2012/02/08/world/asia/japan-older-gamers). La nouvelle génération de personnes âgées est plus familière des jeux d'arcade et des jeux télévisés que leurs homologues de la génération précédente. Les sociétés de machines de jeu sont en train de modifier les jeux d'arcade pour assister la rééducation et promouvoir la santé. Par exemple, «Drum Master» est un jeu qui consiste à comparer le rythme correct de frappe de la batterie dans les chansons. Les versions modifiées sorties en 2004 ont intégré des chansons qui sont plus familières aux personnes âgées, ont ajouté des mécanismes d'assistance à la préhension des manettes, ont adapté la position de la batterie aux fauteuils roulants et adopté des critères de ryhtme de frappe plus faciles. La même année, cette société a créé des institutions de services de soins qui se sont équipées de machines de jeux. Aujourd'hui, différents types de machines de jeux pour l'entraînement physique et mental sont vendus pour les personnes âgées. Ces machines sont destinées à faire office d'alternative au personnel de rééducation et à réduire les coût des soins et de la rééducation.

\section{Conclusion}

Au cours des dernières décennies, de nombreuses technologies pour les personnes âgées ont été développées et vendues. Certaines sont toujours utilisées et continuent d'être modifiées, d'autres ont 
perdu en popularité. L'auteur suppose que les clés de la popularité sont les trois facteurs suivants : moins ennuyant pendant l'utilisation, assistance mimimum pour ne pas perdre la fonction et adapté à l'époque. Les personnes âgées évaluent très soigneusement les bénéfices et les risques de l'utilisation de la technologie. Cette année, une société de téléphones mobiles a commencé à vendre un robot humanoïde de communication (http://www.softbank.jp/en/robot/) directement aux consommateurs. Comme mentionné auparavant, les robots de communication sont destinés à être une plateforme pour de multiples services fournis aux personnes âgées. Le gouvernement japonais a établi la stratégie robotique du Japon (http://www.meti.go.jp/english/press/2015/pdf/0123_01b.pdf) et fait fortement avancer cette technologie pour l'industrie future. Si tous les besoins nécessaires, la compensation, la communication et la promotion de la santé sont intégrés dans des robots, cela constituera une vraie révolution technologique qui garantira la réussite d'une société super-vieillissante.

\section{Références :}

Muramatsu, N. \& Akiyama, H. (2011) Japan: Super-Aging Society Preparing for the Future. The Gerontologist, $51 / 4,425-432$.

Hougaard S., Ruf S. \& Egger C. (2013) EuroTrak + JapanTrak 2012: Societal and Personal Benefits of Hearing Rehabilitation with Hearing Aids - http://www.hearingreview.com/2013/03/eurotrak-japantrak-2012societal-and-personal-benefits-of-hearing-rehabilitation-with-hearing-aids/\#sthash.9spe5nAY.dpuf.

\section{Remerciements :}

Les informations en anglais accessibles via Internet ont été principalement citées pour les lecteurs non natifs japonais. Cet article a bénéficié d'une aide 263101040 du Ministère de l'Éducation, de la culture, des sports, des sciences et de la technologie du Japon. L'âge moyen des DJ a été fourni par Miss Kazuko Yasumara.

Auteur : Prof. Dr. Yasuyuki Gondo est Professeur associé à l'université d'Osaka, Graduate School of Human Sciences and Clinical Thanatology and Geriatric Behavioral Science.

Contact : ygondo@hus.osaka-u.ac.jp. 